\title{
El acoso sexual en contra de las mujeres en escenarios masculinizados, análisis teórico desde el feminismo y el constructivismo estructuralista
}

Sexual harassment against women in masculinized scenarios, theoretical analysis from feminism and structuralist constructivism

Hugo Martínez Ochoa ${ }^{1}$

\begin{abstract}
Resumen
El presente trabajo analiza desde la postura teórica feminista y constructivista estructuralista la situación del acoso sexual que sufren las mujeres en espacios masculinizados. Para ello, utilizo la conceptualización de patriarcado desde Gerda Lerner, género en Joan Scott, la diferencia sexual mediante Marta Lamas, acoso sexual en Stanley y Wise, acosos horizontales y verticales desde Nancy Fraser, acoso sexual ambienta por Ronda, interseccional desde la visión de Crenshaw y el continuum de violencia mediante Liz Kelly. Desde el constructivismo - estructuralista con los conceptos: dominación y violencia simbólica a través de Bourdieu y el término de folklore por Gramsci.
\end{abstract}

Palabras clave: acoso sexual, feminismo, patriarcado, género y constructivismo estructuralista.

\begin{abstract}
This paper analyzes the situation of sexual harassment suffered by women in masculinized spaces from the feminist theoretical and structuralism constructivist position. For this, I use the conceptualization of patriarchy from Gerda Lerner, gender in Joan Scott, sexual difference through Marta Lamas, sexual harassment in Stanley and Wise, horizontal and vertical harassment from Nancy Fraser, sexual harassment by Ronda, intersectional from the

1 Estudiante de Maestría en Acción Pública y Desarrollo Social por el Colegio de la Frontera
Norte, sede Ciudad Juárez. Dirección postal: 32599. Correo electrónico:
hugo_mar24@hotmail.com
\end{abstract}




\section{DOXA}

pISSN: 2395-8758

eISSN: en trámite

vision of Crenshaw and the continuum of violence through Liz Kelly. From the constructivism - structuralism with the concepts: domination and symbolic violence through Bourdieu and the term of folklore by Gramsci.

Keywords: sexual harassment, feminism, patriarchy, gender and structuralism constructivism.

\section{Introducción}

En la presente introducción me gustaría dejar claro a qué me refiero cuando digo en contra de las mujeres; en palabras de Scott (2008), el prefijo: contra; tiene como característica la intencionalidad de dañar, por lo tanto, cuando la violencia se instaura del patriarcado vs mujeres, existe una intencionalidad de dominio hacia la mujer. Por ello, la situación del contra, la entiendo como un manifiesto de violencia voluntaria en contra de las mujeres.

Por escenarios masculinizados, retomo a Fraser (1997), quien hace alusión a que son aquellos espacios en donde existe una mayoría de hombres, derivado de esto, el contexto colectivo se crea y reviste mediante una conducta uniforme masculinizada en relación con el patriarcado, a través del dominio del entorno total.

Sin embargo, en los escenarios masculinizados, en ocasiones existen mujeres que trabajan, mismas que sufren las violencias del grupo masculino, que van desde la percepción salarial menor ${ }^{2}$ hasta los problemas del acoso e incluso violación sexual.

Por lo anterior, considero pertinente realizar un análisis teórico, desde las corrientes del feminismo y el constructivismo estructuralista; para así, poder acercarme un poco a un análisis sobre los contextos de violencia que sufren las mujeres en dichos espacios en el manifiesto de acoso sexual.

\section{Feminismos y estructuras de dominio}

El análisis del patriarcado es un elemento esencial en la construcción de

2 A pesar de realizar el mismo trabajo que un compañero hombre. 
pISSN: 2395-8758

eISSN: en trámite

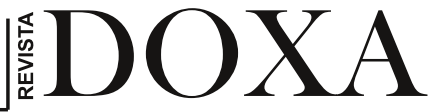

estudios feministas, con esta categoría, se logra comprender la relación de las mujeres con la historia. Para Lerner (1990), el patriarcado explica la naturaleza de la subordinación femenina, las causas de cooperación, las condiciones de resistir ante la misma, el nacimiento del feminismo y la conciencia de que las mujeres han quedado olvidadas en la historia.

Cabe destacar que el sistema patriarcal funciona bajo posturas como la inculcación de los géneros, la privación de la enseñanza, la dependencia económica, la maternidad forzada, la violencia física y psicológica, entre otros factores claves para su estructuración patriarcal.

Provocando así, las condiciones de que el dominado tenga la necesidad de cambiar su situación de sumisión por protección y trabajo no remunerado por manutención (Lerner, 1990). El patriarcado entonces, es un sistema que se establece a través de las desigualdades de género.

Ante esto, Scott (2008), menciona que el género es uno de los elementos centrales para el análisis social, se ha convertido en un sinónimo de la diferencia entre los sexos. Habría que decir también que funge como concepto, categoría, conocimiento, comportamiento, entre otros factores relevantes de la identidad de las personas.

Lamas (1999) dice que al género lo define la acción simbólica -colectiva, mediante la constitución de un orden simbólico de ideas centradas en ser hombre o mujer. Esto quiere decir que el ámbito social es más que un territorio; es un espacio simbólico definido por la imaginación colectiva y determinada en la construcción de la autoimagen de cada persona, es decir, a través de la conciencia habitada por el discurso social.

A lo cual, el género figura como conocimiento. Respecto al conocimiento, Joan Scott (2008) menciona que el género "significa conocimiento de la diferencia sexual" (Scott, 2008, p. 20). Este conocimiento, hace referencia al sentido de la comprensión, que generan las sociedades de cada cultura sobre las relaciones humanas entre hombres y mujeres.

Dicho conocimiento es relativo y se produce bajo formas complejas, en marcos epistemológicos amplios y con una historia autónoma, esto quiere decir que por medio del género, se constituyen actividades a realizar. A saber: comportamientos, prejuicios, construcción de ideologías a partir del cuerpo que se habita.

En contexto con lo anterior, la diferencia sexual no es la causa genealógica de la organización social. Por el contrario, la participación en la historia de la 


\section{DOXA}

pISSN: 2395-8758

eISSN: en trámite

instauración patriarcal, es como se construyó el género hasta nuestros tiempos. Las relaciones sociales y la identificación del género, así como sus roles, son consecuencia de la dominación patriarcal.

Por esto, la producción del conocimiento cultural se da por medio de las desigualdades sociales atribuidas entre hombres y mujeres. A grandes rasgos "el género es una categoría social impuesta a un cuerpo sexuado. El término permite diferenciar la práctica sexual de los roles sociales asignados a las mujeres y a los hombres" (Scott, 2008, p. 53).

Lo expresado hasta aquí supone que la condición de género se manifiesta con la distribución de poder desigual, lo cual crea lazos de dominio en contra de la mujer; uno de los vértices de la violencia suele ser la diferencia sexual y los significados que se le asignan al mismo.

Lamas (2000) menciona que la diferencia sexual puede ser entendida como la sexuación de los seres humanos producida por la simbolización de cada cultura y tiene como resultado las referencias comunes sobre sus actividades y la forma de hacerlas.

Como tal, la situación de la diferencia sexual, determinada por las culturas y el eje patriarcal, tiene como consigna asignar los roles de trabajo, comportamiento, actitudes, entre otros comportamientos; de acuerdo con el cuerpo sexuado que se ocupa.

Ya con anterioridad, Bourdieu (1997a), mencionaba que la diferencia sexual se manifiesta como una operación simbólica que otorga el significado a los cuerpos de las mujeres y los hombres. Bajo este esquema, se hace un reflejo de la realidad y se categoriza como normal, formando un hábito, que se convierte en producto histórico y cultural.

Con lo señalado anteriormente por Bourdieu, se hace visible que todas las actividades que históricamente han pertenecido al hombre, suelen tener una desacreditación si una mujer habita el cuerpo de dicha actividad.

Por lo tanto, no es ajeno que existan críticas hacia las mujeres que se enlistan en trabajos masculinizados. Siendo estas actividades, de acuerdo con Fraser (1997), actividades laborales de los patriarcas.

Es decir, la mujer que habita trabajos masculinizados, se enfrenta a problemas cotidianos como: críticas en el área laboral, menor probabilidad de un ascenso, acosos laborales, acosos sexuales, realizar su trabajo anexo con el doméstico y resistir o aceptar la violencia de la división del trabajo, ya sea por la reproducción patriarcal de su compañero, superior jerárquico $\mathrm{u}$ 
pISSN: 2395-8758

eISSN: en trámite

hombres desconocidos que tienen relación con lo laboral.

Los conceptos y descripciones anteriores, esclarecen que la diferencia sexual y los significados que le asignan a los cuerpos, tienen una relación directa con la violencia que sufren las mujeres en el ámbito laboral.

A este respecto, la mujer que tiene un empleo en donde la mayoría son hombres y se realizan actividades asignadas desde la diferencia sexual a favor del hombre, como son las instituciones policiacas, trabajos en la obra, despachos de abogados, entre otros; existe una mayor incidencia de sufrir violencias desde sus diferentes manifestaciones.

Una de ellas, es el acoso sexual, que suele darse en la cotidianidad y como representación de poder sexual; a este acontecimiento, Stanley y Wise (1992), manifiestan que es una conducta de violencia mayormente contra la mujer, que es parte de un problema social agudo; es una opresión constante y no existe una visibilización real del problema.

Sin embargo, suele ir vinculada y percibida como una conducta socialmente aceptada, a partir del ejercicio del patriarcado, puesto que el hombre considera que tiene el derecho de realizar algún piropo o invitaciones reiteradas a salir, teniendo la meta de un sí como respuesta.

Por medio de los estudios feministas se visualizó al acoso sexual como un problema, debido a que las feministas reconocieron que las conductas del acoso de los hombres hacia las mujeres seguían un patrón y eran legitimadas por la sociedad patriarcal, lo que provocó una coherencia, que culturalizó la manifestación conductual del acoso como una práctica cotidiana.

Respecto a esto, el acoso sexual en el ámbito laboral, se manifiesta como:

"Una conducta masculina indeseada y no correspondida que afirma el rol sexual de una mujer por encima de su función como persona y como trabajadoras y se estima que incluye actos de mirar, comentar o tocar el cuerpo de una mujer, las proposiciones relativas a conductas sexuales o citas amorosas y en última instancia la violación" (Stanley y Wise, 1992, p. 59).

En otro orden de ideas, el acoso se entiende como una conducta donde se utiliza la construcción de género del hombre y la mujer, para lograr poder, o como un acoso en donde se utiliza el poder para obtener sexo o simplemente para manifestar la superioridad del hombre en contra de la mujer.

Por lo tanto, debe ser entendido como una conducta de poder (Stanley \& 


\section{DOXA}

pISSN: 2395-8758

eISSN: en trámite

Wise, 1992). De modo que, el acoso sexual es el resultado de la interacción social desigual entre los géneros.

En síntesis, cuando los hombres disciernen su versión de la realidad, a través de los anteojos del dominio, piensan que cuando su autoridad está en peligro tienen que actuar, dicha actuación está integrada por las diferentes violencias en contra de la mujer, y una de ellas es el acoso sexual, y "sirve para mantener a las mujeres, y a los hombres en los respectivos lugares: en los que los hombres definen como apropiados" (Stanley \& Wise, 1992, p. 93).

El acoso sexual tiene varias formas de presentarse, las cuales toman en cuenta las relaciones de jerarquía entre el hombre y la mujer y los espacios en los cuales esta conducta nociva se perpetra. A este respecto, Fraser (1997), define al acoso sexual horizontal, como aquel que se da en lugares como el trabajo, escuela o cualquier escenario donde la mujer se encuentre en contacto continuo con personas para realizar una actividad.

Los protagonistas del acoso sexual horizontal, suelen ser los compañeros de trabajo, son los hombres quienes, aunque las mujeres no se encuentran laboralmente bajo su autoridad, consideran que pueden acosarlas, simplemente porque ellos son los hombres y ellas las mujeres. "Este tipo de acoso es frecuente cuando un reducido número de mujeres ingresa en ocupaciones dominadas fuertemente por los hombres y masculinizadas" (Fraser, 1997, p. 120).

Por su parte, el acoso sexual vertical, es también una de las variables de acoso presentes en escenarios fuertemente dominado por hombres $y$ masculinizados. Dicha conducta, es perpetrada por los superiores, jefes, supervisores o cualquier hombre que tenga una superioridad jerárquica en un ambiente de trabajo, escuela o institución pública o religiosa.

"El efecto de esta práctica es preservar el control social o de clase que los primeros ejercen sobre los segundos" (Fraser, 1997, p. 120). En otro orden de ideas, el acoso sexual vertical, se suscita como esa conducta de poder en contra de las mujeres, mediante la subordinación logística pero también una subordinación sexual (Fraser, 1997).

Continuando con el análisis, el acoso sexual ambiental, es el tercer tipo. Proviene de compañeros de trabajo (con los cuales no se interactúa comúnmente, sin embargo, se encuentran bajo la misma institución laboral, pública u organización cualquiera) o de terceras personas relacionadas con el trabajo pero que son desconocidas. 
pISSN: 2395-8758

eISSN: en trámite

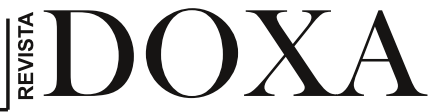

Este tipo de acosos se manifiestan principalmente por parte de hombres desconocidos que tienen relación con el trabajo, compañeros de trabajo con los cuales no se interactúa y, por último, con personas desconocidas que no tienen nada que ver con las labores (Ronda, 2009).

A su vez, el acoso sexual no es solamente una situación de dominación de género, sino también de clase, posición social y racial; con esto quiero decir que a las mujeres se les suele acosar no sólo para mantener una relación sexual forzada, sino también para reafirmar el poder del hombre sobre la mujer.

A esto Crenshaw (1991), indaga que la interseccionalidad es un fenómeno por el cual, las mujeres sufren violencias múltiples a partir de las categorías sociales de situación política, clase social, pertenencia racial, edad, creencias e ideologías.

Las mujeres que se encuentran en una situación de vulnerabilidad por ser de clase social baja, latina, afrodescendiente, ser muy joven o una adulta mayor, con una ideología política contraria a la establecida, entre otros factores; sufren violencias que las mujeres blancas, clase media, clase alta, estado migratorio legal e ideologías a favor del gobierno en turno, no sufren.

Tales violencias suelen ser: discriminación racial, deportación migratoria, violencia psicológica y física por su condición racial, acoso sexual para la posesión sexual, abuso sexual y feminicidio.

En el punto particular del acoso sexual como violencia interseccional, es sabido que es una conducta que afecta a todas las mujeres, sin embargo, esas afectaciones son distintas dependiendo de muchos factores sociales, tales como la situación política, migración, económica, cultural, racial, edad, creencias, ideologías, entre otros factores, que son parte esencial de las culturas y los cuerpos de las mujeres.

Es decir, la violencia del acoso sexual en contra de las mujeres en este sentido, hace que la vivencia de la dominación masculina sea diferente experiencia de violencia sexual.

Me refiero a que, para algunas mujeres puede ser el acoso sexual una barrera para el desarrollo laboral (por no acceder a una cita con quien ejerce el acoso), falta de recursos económicos (por negarse al piropo o la relación sexual), e incluso problemas de depresión y suicidio (debido a comentarios constantes lascivos en contra de una mujer diciéndole que no es atractiva o que por su color de piel nunca tendrían sexo con ellas, entre otras 


\section{DOXA}

pISSN: 2395-8758

eISSN: en trámite

situaciones). En otras palabras, la violencia del acoso sexual se manifiesta en estas posturas y condiciones llamadas violencias interseccionales (Kelly, 1988).

Cabe señalar que la interseccionalidad, ha logrado hacer el análisis de que el acoso sexual, no tiene siempre la finalidad de la copulación, sino que también tiene el objetivo de la posesión sexual, entendiendo posesión sexual como tener el cuerpo de la mujer como cosificación o producto de propiedad al servicio del hombre como ese vínculo de subordinación de las mujeres afrodescendientes y latinas.

A grandes rasgos, es una forma de perpetuar el poder de los hombres anglosajones, afrodescendientes y latinos; en contra de las mujeres en sus diferentes culturas, clases sociales, etnicidades y circunstancias ideológicas.

Entonces, el análisis de la interseccionalidad se da mediante tres elementos de subordinación, la interseccionalidad estructural, la interseccionalidad política y la interseccionalidad racial (Crenshaw, 1991).

La interseccionalidad y sus características forman parte de las violencias de género en contra de las mujeres, a este respecto, existe un continuum de violencia, el cual, explica Kelly (1988), es manifestado a lo largo de los años, bajo diferentes posturas, entes, modos y formas.

La violencia no es desviada, episódica o extraordinaria. Por el contrario, es una violencia que se vive en la cotidianidad bajo conductas no visibles regida por el patriarcado y las construcciones de género. Dicha violencia es normativa y funcional; existe en un contexto cotidiano para las vidas y experiencias de las mujeres alrededor del mundo.

Asimismo, las violencias se mezclan entre sí, sin claridad para lograr la demarcación. Dicho de otra manera, las diferentes manifestaciones de eventos, actos y comportamientos; se ubican en un orden de desigualdad de género y dominación; y se mantienen bajo esa dicotomía como una estructura en lo público y lo privado.

Ante lo explicado, vale la pena retomar el hecho de que la idea del continuum, es que la violencia en contra de la mujer se encuentra en todos los escenarios bajo diferentes formas, sin embargo, respecto al acoso sexual, el escenario mayormente se presenta el ámbito laboral, público y en el hogar.

Inclusive, los perpetradores suelen ser los hombres, que tienen superioridad jerárquica ya sea laboral o cultural, a saber, el caso del jefe de trabajo, el esposo o pareja sentimental, pero también por hombres desconocidos (Kelly, 
pISSN: 2395-8758

eISSN: en trámite

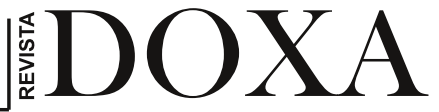

1988).

Retomando lo dicho, la dominación masculina y la violencia simbólica, desde la perspectiva estructuralismo - constructivista, la violencia simbólica funge como un instrumento de la dominación masculina, misma que produce un orden social y funciona como una máquina simbólica que tiende a ratificar la dominación de las actividades asignadas a los géneros, su espacio, momento, instrumentos, identidad y violencia. En otras palabras, la dominación masculina y la violencia simbólica determinan los espacios y conductas, tanto para el hombre como para la mujer.

La dominación masculina, tiene todas las condiciones para que exista su pleno ejercicio en la sociedad. El dominio reconocido universalmente a favor de los hombres se afirma en la objetividad de la estructura social, es decir, destacan las actividades productivas y reproductivas de la división sexual del trabajo, la reproducción biológica y los roles de género asignados a la sociedad derivados del patriarcado, esto provoca los hábitos de la cotidianidad.

Es decir, los esquemas de dominación, funcionan como paradigmas de percepción social, por medio de la formación de capitales de pensamiento y acciones uniformes en la sociedad. Esto quiere decir que se homologa la situación espacial y conductual de los cuerpos del ser hombre o mujer.

Cabe señalar que al hombre le corresponde el espacio público, los trabajos al exterior del hogar, la libertad sexual y la reproducción biológica voluntaria; por parte de las mujeres es todo lo contrario, a ella, le corresponde el espacio privado, los trabajos al interior del hogar, esclavitud sexual y la reproducción biológica forzada.

"En consecuencia, la representación androcéntrica de la reproducción biológica y la reproducción social se ve investida por la objetividad de un sentido común, entendido como consenso práctico y dóxico, sobre el sentido de las prácticas" (Bourdieu, 2000, p. 27-28).

Es decir, que las relaciones de poder androcéntricas y patriarcales, fabrican los roles del hombre y la mujer a partir, de las relaciones de poder que generan la creencia de que no tiene que pensarse ni afirmarse su rol social, sino que solamente tiene que vivirse de acuerdo al género al que se pertenezca y que crea de algún modo la violencia simbólica que la mujer sufre (Bourdieu, 2000).

Respecto a la violencia simbólica que se manifiesta en la dominación

Vol. 8 Núm. 14 Enero - Junio 2018 


\section{DOXA}

pISSN: 2395-8758

eISSN: en trámite

masculina, suele presentarse cuando el dominado se siente obligado a conceder al dominador su condición de vida a partir de los capitales del patriarcado y androcentrismo.

El capital del patriarcado, entendido como un sistema de control en contra de la mujer instaurado por los hombres, que tiene por objetivo la subordinación femenina y al capital androcéntrico como un modelo de centralidad masculina para el ejercicio del poder.

Como tal, la violencia simbólica, se produce a partir del conocimiento de los capitales mencionados y dicho conocimiento se comparte tanto por el dominador como por el dominado y suelen figurar en la relación de dominación como un hecho natural y común.

"La apreciación se da bajo los conceptos de verticalidad alto/bajo, masculino/femenino, blanco/negro; y son el producto de la asimilación de las clasificaciones de naturalización de un ser social" (Bourdieu, 2000, p. 28).

A esto, menciona Bourdieu (1997b) que:

"La violencia simbólica, es esa coerción que se instituye por mediación de una adhesión que el dominado no puede evitar otorgar al dominante $(\mathrm{y}$, por lo tanto, a la dominación) cuando sólo dispone, para pensarlo y pensarse o, mejor aún, para pensar su relación con él, de instrumentos de conocimiento que comparte con él, al no ser más que la forma incorporada de la estructura de la relación de dominación, hacen que ésta se presente como natural" (p. 225).

Como se ha mencionado, la violencia simbólica produce un poder hipnótico sobre la mujer y a través de la manifestación del dominador, se producen conminaciones, sugerencias, seducciones, amenazas, reproches, órdenes para una relación de dominio, masculino en la vida cotidiana.

Cabe destacar que, respecto al efecto de lo cotidiano, es donde se encuentran los esquemas de percepción, evaluación y conducta que fundamentan, las decisiones sobre la dominación masculina hacia las mujeres, los controles de la voluntad, el conocimiento, reconocimiento de las prácticas de dominación, su relación con el género y el poder.

Entonces se podría decir que:

"La dominación masculina está formada por antonomasia de la violencia simbólica, y la sumisión femenina, respecto a la cual cabe decir que es a la vez, y sin contradicción espontánea y extorsionada, si se advierten los efectos duraderos que el orden social ejerce sobre las mujeres, es decir,

76 Vol. 8 Núm. 14 Enero - Junio 2018 
las disposiciones espontáneamente concedidas a este orden que la violencia simbólica les impone" (Bourdieu, 1997b. p. 226).

Es decir, la dominación masculina es el producto de un trabajo del patriarcado y el androcentrismo, bajo la estructura de instituciones como la familia patriarcal, la iglesia, escuela y Estado (Bourdieu, 2000).

Hay que mencionar aparte que en un punto particular la dominación masculina, se hizo visible (no es que no existiera antes) cuando las mujeres entraron al mundo laboral externo en mayor medida, debido a las condiciones de globalización y capitalismo.

Respecto a esto, la dominación tomó nuevas estructuras de instrumentación para perpetrar la violencia en contra de las mujeres. El acoso sexual ha sido uno de los instrumentos mayormente utilizados, teniendo presente que no siempre tiene por objetivo la relación sexual, pero si la posesión como ese círculo de poder de dominación.

El acoso sexual es una herramienta para la dominación mayormente utilizada por los hombres, mediante el ejercicio del deseo masculino de posesión sexual y que lo posiciona por encima socialmente de la mujer.

Es decir, el acoso sexual tiene su cercanía con las condiciones de vida cultural de los territorios. A esto, menciona Gramsci (1999) que el folklore tiene que ser estudiado o utilizado como categoría para los estudios sociales, como aquel concepto que tienen las personas del mundo y de la vida.

Dicha concepción, son los anteojos con los cuales la sociedad, a partir del cuerpo que habitan y las violencias que viven, perciben las conductas ordinarias y las extraordinarias (Gramsci, 1999).

En palabras de este autor:

"El folklore puede ser comprendido sólo como un reflejo de las condiciones de vida cultural del pueblo, si bien ciertas concepciones propias del folklore se prolongan incluso después de que las condiciones son (o parecen) modificadas o dan lugar a combinaciones extrañas" (1999. p. 204).

Esto quiere decir que el folklore es ese modelo de concepción del mundo que tienen los pobladores de un territorio en específico, sin embargo, no es creado desde la individualidad, por el contrario, es creado a partir del sistema del conjunto de clases, el patriarcado, el género, la política, el androcentrismo y es organizado a partir del método dominador - dominado.

Asimismo, el folklore del pueblo crea condiciones estructurales, como lo es 


\section{DOXA}

pISSN: 2395-8758

eISSN: en trámite

la moral del pueblo, en donde, se crean conductas socialmente aceptadas, a partir, de costumbres que derivan de la superstición, el patriarcado, la religión, la política, la pureza y la sumisión de las mujeres.

Por medio del mismo, se gestan conductas morales de acuerdo con el género al que se identifique la persona, esto destaca en el comportamiento que debe de tener cada ente, de acuerdo con el género que pertenezca. Una situación relevante es que, sí las conductas de las mujeres y los hombres se ven rebasadas o van en contra de la moral establecida, se producen discursos sociales en contra de los mismos por actuar de acuerdo con el paradigma de comportamiento.

En un ejemplo práctico, el acoso sexual es una de las prácticas que está socialmente aceptada por la moral del pueblo, la función de la mujer ante el acoso es contestar con una sonrisa, decir gracias o simplemente seguir de paso.

Pero, sí una mujer se defiende mediante pedir respeto, regresar la ofensa con un palabra altisonante o una agresión física, la lleva a instalarse en una inmoralidad social, por lo cual es perseguida por el folklore, señalándola como muchacha irrespetuosa, apretada, mala mujer o incluso como prostituta o mujerzuela. El folklore como tal, está construido a favor de los hombres para el libre ejercicio de la violencia en contra de la mujer (Gramsci, 1999).

\section{Conclusiones}

El patriarcado ha inculcado por medio de los géneros la acción simbólica colectiva del comportamiento, misma que desde la dominación ha generado el orden de ser hombre o mujer y a su vez ha producido la diferencia sexual a través de la ventaja del hombre sobre la mujer.

Teniendo con ello la ocupación del espacio público y el control del espacio privado, mismo en donde han depositado a las mujeres. Sin embargo, con los nuevos modelos de producción laboral, la necesidad económica y los nuevos espacios de producción, el patriarcado ha visto la necesidad de que la mujer labore fuera del hogar.

Por ello, surgió un cuestionamiento de la dominación: ¿Qué hacemos para seguir dominando y controlando a las mujeres en el espacio público? Uno de los nuevos mecanismos fue el acoso sexual. Entendido como una conducta de poder que afirma el rol sexual de una mujer por encima de su función como 
trabajadora.

El acoso sexual ejercido en el ámbito laboral ha tenido dos funciones, por una parte la posesión como método de violencia discriminatoria, que tiene por objetivo el doblegar a las mujeres y discriminarlas por medio de su posición social, política, género, racial y estética; ello por medio de agresiones verbales, despidos, burlas, entre otros.

Y por otra parte el acoso sexual con fines sexuales o de copulación. El mismo es ejercido tanto por superiores, compañeros y hombres extraños con los cuales interactúan por la labor. Ambos acosos se presentan en un continuum de violencia que tiene la finalidad del control de la mujer en los espacios laborales y la reafirmación del patriarcado en sus cuerpos y voluntades.

A lo anterior, desde la interpretación estructuralista - constructivista, la conducta del acoso sexual se expresa mediante la dominación masculina a partir de las actividades productivas, reproductivas, de la división sexual del trabajo y los roles de género asignados por la dominación. Es en ese momento donde se produce el piropo, las invitaciones recurrentes a salir, la intención de tocar, la burla, los tocamientos, entre otras conductas.

$\mathrm{Y}$ en un segundo momento, a partir de la violencia simbólica, la mujer se siente obligada a conceder al dominador una respuesta pasiva, a partir de su conocimiento de los capitales del patriarcado y el androcentrismo. Mismos que gestan la aceptación del acoso porque no existe una forma efectiva de enfrentarlo.

Esto debido a que el acoso es aceptado mediante el folklore del pueblo; el acoso no es siquiera un problema. Por el contrario, es sólo una manifestación de ser hombre y desde esa construcción es una violencia invisible que tiene la capacidad de controlar, dañar, e incluso estar implicada como colaboradora de abusos sexuales, violaciones e incluso feminicidios.

\section{Referencias}

Bourdieu, P. (1997). Meditaciones Pascalianas. España: Anagrama.

Bourdieu, P. (1997). Razones prácticas, sobre la teoría de la acción. España: Anagrama.

Bourdieu, P. (2000). Dominación masculina. México: Anagrama.

Crenshaw, K. W. (1991). Mapping the margins: intersectionality, identity politics, and violence against women of color. Stanford Law

Vol. 8 Núm. 14 Enero - Junio 2018 
Review, 1241-1299.

Fraser, N. (1997). Justicia Interrupta. reflexiones críticas desde la reflexión postsocialista. Colombia: Siglo del Hombre Editores y Universidad de los Andes.

Gramsci, A. (1999). Cuadernos de la cárcel, Tomo 6 (Cuaderno 27 "Observaciones sobre el folklore"). México: Biblioteca Era.

Kelly, L. (1988). Surviving Sexual Violence. United State: University of Minnesota Press.

Lamas, M. (1999). Usos, dificultades y posibilidades de la categoría género. págs. 1-23.

Lamas, M. (2000). Diferencias de sexo, género y diferencia sexual. Cuicuilco, $1-24$.

Lerner, G. (1990). La creación del patriarcado. Barcelona: Crítica.

Ronda, C. P. (2009). Guía sindical Violències de gènere a l'empresa. Barcelona, España: Secretaria de la Dona de CCOO de Catalunya.

Scott, J. W. (2008). Género e Historia. México: Fondo de Cultura Económica.

Stanley, L., \& Wise, S. (1992). El acoso sexual en la vida cotidiana. España: Paidós. 